\title{
circARRDC3 contributes to interleukin-13-induced inflammatory cytokine and mucus production in nasal epithelial cells via the miR-375/KLF4 axis
}

\author{
TAO WANG ${ }^{1-3}$, PEIHUA WANG ${ }^{1-3}$, DONG CHEN ${ }^{1-3}$, ZHOU XU $^{1-3}$ and LIYUN YANG ${ }^{1-3}$ \\ ${ }^{1}$ Department of Otolaryngology Head and Neck Surgery, Shanghai Ninth People's Hospital, School of Medicine, \\ Shanghai Jiao Tong University, Shanghai 200011; ${ }^{2}$ Ear Institute Shanghai Jiaotong University School of Medicine; \\ ${ }^{3}$ Shanghai Key Laboratory of Translational Medicine on Ear and Nose Diseases, Shanghai 200125, P.R. China
}

Received December 3, 2019; Accepted October 30, 2020

DOI: $10.3892 / \mathrm{mmr} .2020 .11780$

\begin{abstract}
Allergic rhinitis (AR) is a common inflammatory disorder of the nasal mucosa. It is a major risk factor for asthma development, and uncontrolled AR can lead to the worsening of asthma symptoms, which affects the quality of life and productivity of patients. Circular RNAs (circRNA) were reported to be involved in the pathogenesis of AR. The aim of the present study was to investigate the functional role of circRNA arrestin domain-containing 3 (circARRDC3) in AR progression. circARRDC3 knockdown suppressed the levels of granulocyte-macrophage colony-stimulating factor (GM-CSF) and eotaxin and mucin 5AC (MUC5AC) in IL-13-induced nasal epithelial cells. Moreover, circARRDC3 silencing promoted viability and suppressed apoptosis in IL-13-induced NECs. circARRDC3 targeted microRNA (miR)-375 and negatively regulated its expression. miR-375 inhibition reversed the effects of circARRDC3 knockdown on GM-CSF, eotaxin and MUC5AC expression levels, cell viability and cell apoptosis. In addition, miR-375 inhibited krueppel-like factor 4 (KLF4) expression through direct interaction, and miR-375 overexpression inhibited GM-CSF, eotaxin and MUC5AC expression levels, and cell apoptosis, which was abolished following KLF4 overexpression. In addition, circARRDC3, miR-375 and KLF4 were all dysregulated in the nasal mucosa of patients with AR. miR-375 expression was negatively correlated with circARRDC3 and KLF4 expression, and circARRDC3 expression was positively correlated with KLF4 expression. In conclusion, circARRDC 3 contributed to the development of
\end{abstract}

Correspondence to: Dr Peihua Wang, Department of Otolaryngology Head and Neck Surgery, Shanghai Ninth People's Hospital, School of Medicine, Shanghai Jiao Tong University, 639 Zhizaoju Road, Shanghai 200011, P.R. China

E-mail: peihuawang366@163.com

Key words: allergic rhinitis, circular RNA arrestin domain-containing 3, microRNA-375, Krueppel-like factor 4
AR by regulating the miR-375/KLF4 axis. These findings may provide novel insights into the pathogenesis of AR.

\section{Introduction}

Allergic rhinitis (AR) is a chronic inflammatory disorder of the nasal mucosa, which affects $10-30 \%$ of the worldwide population (1). There are two types of AR: Seasonal and perennial (2). Both types of AR typically manifest with pruritus, sneezing, rhinorrhea and nasal congestion (3). Inherited genetics and environmental exposure contribute to the development and progression of AR, which frequently leads to asthma (4). Considering the comprehensive pathogenesis, AR is often misdiagnosed and patients develop a resistance to drug treatments. Therefore, there is an urgent need to determine the molecular mechanisms underlying the progression and development of AR.

Circular RNAs (circRNAs) are a novel class of non-coding RNAs characterized by covalently closed-loop structures (5). Several studies have indicated that circRNA dysregulation is involved in inflammatory-related diseases, including AR. For example, Liu and Cao (6) reported that circDdx17 downregulated microRNA (miR/miRNA)-17 expression and alleviated AR symptoms and pathological conditions. Zhu et al (7) indicated that circHIPK3 overexpression accelerated $\mathrm{T}$ helper cell (Th) 2 differentiation of ovalbumin-induced CD4 ${ }^{+} \mathrm{T}$ cells and aggravated the nasal symptoms of AR mice. However, the exact function of circRNA arrestin domain-containing 3 (circARRDC3) in the progression of AR remains unknown.

Granulocyte-macrophage colony-stimulating factor (GM-CSF) and eotaxin are pro-inflammatory cytokines in allergic airway inflammation that are synthesized and released by airway epithelial cells, infiltrating leukocytes and fibroblasts in response to allergens and inflammatory mediators (8). Mucus hypersecretion, particularly mucin 5AC (MUC5AC) expression, is a common feature of allergic airway disorders (9). Accumulating studies have indicated that the inflammatory factors GM-CSF, eotaxin and MUC5AC are indicators of AR $(8,10)$. Therefore, the aim of the present study was to evaluate the relationship between circARRDC3 and GM-CSF, eotaxin or MUC5AC. 
miRNAs are a type of short non-coding RNAs with a length of $\sim 22$ nucleotides $(11,12)$, which have been implicated in the pathogenesis of AR. For instance, Teng et al (13) demonstrated that miR-143 suppressed IL-13-induced inflammatory cytokine production by targeting IL-13 receptor $\alpha 1$ in AR. Liu et al (14) reported that miR-487b ameliorated AR via inactivation of the IL-33/sulfotransferase signaling pathway. Chen et al (15) indicated that miR-21 was associated with antenatal immunoglobulin E production and development of AR. Furthermore, miR-375 has been reported to play an inhibitory role in the development of allergic inflammation (16). Nevertheless, the biological role of miR-375 in AR needs to be further elucidated.IL-13, a typical Th2 cytokine, is involved in mucus hypersecretion and the release of inflammatory mediators by airway epithelial cells (17). A growing number of studies focused on preventing or reducing allergic airway reactions by targeting IL-13. For instance, Lv et al (18) indicated that IL-37 attenuated IL-4/IL-13-induced eotaxin production and lung eosinophilia in murine allergic asthma. Ashraf et al (19) reported that oxyresveratrol could improve allergic asthma by downregulating the expression levels of IL-4, IL-5 and IL-13. Since IL-13 is closely related to the pathogenesis of allergic diseases, the IL-13 induction model has been widely used in AR research.

The aim of the present study was to investigate the role of circARRDC3 in IL-13-induced nasal epithelial cells (NECs). The results indicated that circARRDC3 contributed to IL-13-induced inflammatory cytokine and mucus production in NECs via the miR-375/krueppel-like factor 4 (KLF4) axis.

\section{Materials and methods}

Tissue collection. Nasal mucosa samples from the inferior turbinate mucosa were obtained from 20 healthy subjects

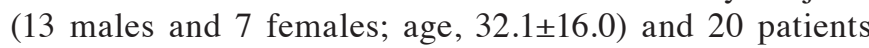
with AR. (males and 9 females; age, 36.4+13.8). Samples were stored at $-80^{\circ} \mathrm{C}$ until further use. The present study was approved by The Ethics Committee of The Shanghai Ninth People's Hospital (Shanghai, China). Written informed consent was obtained from all patients.

Cell culture. Under local anesthesia, inferior turbinate nasal epithelial cells (NECs) were obtained using the nasal fragment method (20). Cells were cultured in bronchial epithelial cell growth medium (BEGM; Lonza Group, Ltd.) with 10\% FBS (Gibco; Thermo Fisher Scientific, Inc.) in a humidified 5\% $\mathrm{CO}_{2}$ incubator. The NECs were passaged when a 70-80\% fused monolayer was present. Cells from passage 2 were used for subsequent experiments.

Cell transfection. Small interfering RNA (siRNA) targeting circARRDC3 (si-circARRDC3) and negative control (si-NC), miR-375 mimic and negative control (NC mimic), and miR-375 inhibitor and negative control (NC inhibitor) were purchased from Shanghai GenePharma Co., Ltd. circARRDC3 or KLF4 cDNA was amplified and subcloned into pcDNA3.1 vectors (Shanghai GenePharma Co., Ltd.) to overexpress circARRDC3 or KLF4. pcDNA3.1 empty vector (pcDNA3.1) as a control.

All plasmids $(0.5 \mu \mathrm{g})$ and oligonucleotides $(50 \mathrm{nM})$ were transfected into NECs cells $\left(6 \times 10^{5}\right.$ cells/well) using
Lipofectamine $^{\mathrm{TM}} 2000$ (Thermo Fisher Scientific, Inc.). After transfection for $48 \mathrm{~h}$, the cells were used for subsequent assays. The sequences of the oligonucleotides used for transfection were as follows: i) si-circARRDC3, 5'-CAGACCTCATCACTCA GAA-3'; ii) siNC, 5'-CCGAAGGCCGTGTCCCGAG-3'; iii) miR-375 mimic, 5'-UUUGUUCGUUCGGCUCGCGUGA-3'; iv) NC mimic, 5'-UUUGUACUACACAAAAGUACUG-3'; v) miR-375 inhibitor, 5'-UCACGCGAGCCGAACGAACAAA-3'; and vi) NC inhibitor, 5'-CAGUACUUUUGUGUAGUACAAA-3'.

IL-13 stimulation of NECs. Following lentivirus infection, NECs $\left(0.75 \times 10^{5} /\right.$ well $)$ were stimulated with $50 \mathrm{ng} / \mathrm{ml} \mathrm{IL-13}$ (R\&D Systems, Inc.) for 14 days at $37^{\circ} \mathrm{C}$ in BEGM without hydrocortisone. The media was replaced twice per week. Cell supernatant and pellets were collected centrifuged for $3 \mathrm{~min}$ at $4^{\circ} \mathrm{C}$ for reverse transcription-quantitative PCR (RT-qPCR).

MTT assay. Transfected cells were seeded into 96-well culture plates $\left(2 \times 10^{4}\right.$ cells/well). A total of $\sim 20 \mu 1$ MTT solution was added to each well, and the cell culture plates were incubated at $37^{\circ} \mathrm{C}$ for $4 \mathrm{~h}$. DMSO $(150 \mu \mathrm{l})$ was added following supernatant removal. The absorbance was determined at a wavelength of $490 \mathrm{~nm}$ using a microplate reader (Bio-Rad Laboratories, Inc.).

Luciferase reporter assay. The Starbase (version 2.0; http://starbase.sysu.edu.cn/starbase2/) and TargetScan (Release 7.2; http://www.targetscan.org/vert_72/) online tools were used to predict the downstream targets of circARRDC3 and miR-375, respectively (21). Wild-type and mutant circARRDC3 or KLF4 sequences were subcloned into the pmirGLO vector (Promega Corporation). Subsequently, the vectors were co-transfected with miR-375 mimic, NC mimic, miR-375 inhibitor or $\mathrm{NC}$ inhibitor into $293 \mathrm{~T}$ cells $\left(2 \times 10^{5}\right.$ cells/well) using Lipofectamine ${ }^{\circledR} 2000$ (Thermo Fisher Scientific, Inc.). Following $48 \mathrm{~h}$ of transfection, a dual-luciferase reporter system (Promega Corporation) was used to measure luciferase activity. Firefly luciferase activity was normalized to Renilla luciferase.

$R T-q P C R$. Total RNA was extracted from NECs or nasal mucosal specimens using TRIzol ${ }^{\circledR}$ reagent (Invitrogen; Thermo Fisher Scientific, Inc.). RT was performed using the PrimeScript Reagent kit (Takara Bio, Inc.) at $37^{\circ} \mathrm{C}$ for $30 \mathrm{~min}$. qPCR was performed using the SYBR-Green PCR Master Mix kit (Takara Bio, Inc.) on the ABI Prism 7300 Sequence Detection system (Applied Biosystems; Thermo Fisher Scientific, Inc.). The thermocycling conditions consisted of an initial denaturation at $95^{\circ} \mathrm{C}$ for $15 \mathrm{sec}$, followed by 40 cycles of denaturation at $94^{\circ} \mathrm{C}$ for $30 \mathrm{sec}$, annealing at $60^{\circ} \mathrm{C}$ for $20 \mathrm{sec}$, and extension at $72^{\circ} \mathrm{C}$ for 40 sec. GAPDH and U6 were used as internal controls. Gene expression was analyzed using the $2^{-\Delta \Delta \mathrm{Cq}}$ method (22). The following primer pairs were used for the qPCR: circARRDC3 forward, 5'-TCCTATTGCTCTTCCTTGTGG-3' and reverse, 5'-AAAAAGTCAGGGCAGCAGAG-3'; miR-375 forward, 5'-AAACCGGACCTGAGCGTTTT-3' and reverse, 5'-CCGAAC GAACAAAACGCTCA-3'; KLF4 forward, 5'-TCTCCCACATG AAGCGACTT-3' and reverse, 5'-ATGGGTCAGCGAATTGG AGA-3'; GAPDH forward, 5'-TCGTGGAAGGACT CATG ACC-3' and reverse, 5'-ATGATGTTCTGGAGAGCCCC-3'; and 
U6 forward, 5'-CTCGCTTCGGCAGCACATA TACTA-3' and reverse, 5'-ACGAATTTGCGTGTCATCCTT GCG-3'; GM-CSF forward, 5'-ATGTGGCTGCAGAGCCTGC TGC-3' and reverse, 5'-CTCCCAGCAGTCAAAGGG-3'; eotaxin forward, 5'-CCC CTTCAGCGACTAGAGAG-3' and reverse, 5'-TCTTGGGGTCG GCACAGAT-3'; MUC5AC forward, 5'-TGATCATCCAGCAGG GCT-3' and reverse, 5'-CCG AGCTCAGAGGACATATGGG-3'.

Western blot analysis. NECs were lysed with pre-cooled RIPA lysis buffer (Beyotime Institute of Biotechnology) and quantified using a BCA Protein Assay kit (Beyotime Institute of Biotechnology). A total of $20 \mu \mathrm{g}$ protein/lane was added into each well of a vertical electrophoresis tank and separated by SDS-PAGE on $10 \%$ gels. Subsequently, the proteins were transferred to PVDF membranes. After blocking with $5 \%$ skim-milk for $1 \mathrm{~h}$ at room temperature, the membranes were incubated with primary antibodies specific for KLF4 (1:1,000; Novous; cat. no. IMG-6081A) and GAPDH antibody (1:500; Santa Cruz Biotechnology, Inc.; cat. no. sc-47724) at $4^{\circ} \mathrm{C}$ overnight. The membranes were washed three times with $0.1 \%$ TBS-Tween-20 (each time $5 \mathrm{~min}$ ), then incubated with goat anti-rabbit/mouse HRP-conjugated secondary antibodies (1:5,000; Abcam; cat. nos. ab205718 and ab205719) at room temperature for $1 \mathrm{~h}$. Protein bands were visualized using the Enhanced Chemiluminescence Plus kit (Thermo Fisher Scientific, Inc.), according to the manufacturer's instructions. Protein bands were quantified using Quantity One software (version 4.6.6; Bio-Rad Laboratories, Inc.).

TUNEL assay. The TUNEL assay was performed using an In Situ Cell Death Detection kit (Roche Diagnostics $\mathrm{GmbH}$ ) according to the manufacturer's instructions. After rising twice by PBS, NECs cells fixed by $4 \%$ formaldehyde for $25 \mathrm{~min}$ at $4^{\circ} \mathrm{C}$. The cells were permeabilized by $0.2 \%$ Triton X-100 for $5 \mathrm{~min}$. The cells were then equilibrated with $100 \mu 1$ Equilibration buffer for $10 \mathrm{~min}$ at room temperature. Subsequently, cells were labeled with $50 \mu 1$ terminal deoxynucleotidyl transferase (TdT) reaction cocktail for $45 \mathrm{~min}$ at $37^{\circ} \mathrm{C}$, followed by treatment with Click-iT reaction cocktail. The nucleus was stained with hematoxylin or methyl green at room temperature for $1 \mathrm{~h}$. A fluorescence microscope (magnification, x100; Olympus Corporation) was utilized to observe TUNEL-positive cells in at least 5 fields of view.

Statistical analysis. Statistical analyses were performed using SPSS 23.0 software (IBM Corp.). Data are presented as the mean $\pm \mathrm{SD}$ from at least three independent experiments. Student's t-test or one-way ANOVA followed by Tukey's post hoc test were used for comparisons between groups. Correlation analysis was carried out using Pearson's correlation coefficient. $\mathrm{P}<0.05$ was considered to indicate a statistically significant difference.

\section{Results}

circARRDC3 regulates $I L-13$-induced inflammatory cytokines, mucus production, cell viability and apoptosis of NECs. To determine the effects of circARRDC 3 on AR development, si-circARRDC3 or pcDNA3.1-circARRDC3 were transfected into NECs to inhibit or overexpress circARRDC3 (Fig. 1A). As shown in Fig. 1B-D, knockdown of circARRDC3 downregulated the levels of GM-CSF, eotaxin and MUC5AC, whereas circARRDC3 overexpression upregulated the levels of GM-CSF, eotaxin and MUC5AC. In addition, circARRDC3 interference significantly promoted the viability of IL-13-treated NECs, whereas circARRDC3 overexpression significantly repressed cell viability (Fig. 1E). Furthermore, knockdown of circARRDC3 significantly suppressed the apoptosis of IL-13-treated NECs, and the addition of circARRDC3 significantly promoted cell apoptosis (Fig. 1F). Collectively, the data indicated that circARRDC3 may contribute to AR development.

miR-375 interacts with circARRDC3. Based on bioinformatics analysis (StarBase 2.0), miR-375 was predicted to be a downstream target of circARRDC3 (Fig. 2A). RT-qPCR indicated that the expression of miR-375 was upregulated in NECs transfected with miR-375 mimic and was downregulated in NECs transfected with miR-375 inhibitor (Fig. 2B). As shown in Fig. 2C, transfection with the miR-375 mimic decreased and miR-375 inhibitor increased the luciferase activity of circARRDC3-WT, while no significant difference was observed in the circARRDC3-Mut group. To determine whether miR-375 was critical for circARRDC3-mediated AR development, si-NC, si-circARRDC3 and si-circARRDC3 + miR-375 inhibitor were transfected into IL-13-treated NECs. RT-qPCR revealed that knockdown of circARRDC3 significantly upregulated miR-375 expression, which was reversed following miR-375 inhibitor transfection (Fig. 2D). Moreover, transfection with the miR-375 inhibitor reversed the inhibitory effects of circARRDC3 knockdown on the levels of GM-CSF, eotaxin and MUC5AC in IL-13-induced NECs (Fig. 2E-G). Further results indicated that circARRDC3 knockdown promoted the viability of IL-13-induced NECs, whereas the effects were reversed by miR-375 inhibition (Fig. 2H). Furthermore, the depletion of circARRDC3 suppressed the apoptosis of IL-13-induced NECs, which was abolished following miR-375 inhibitor transfection (Fig. 2I). In summary, these results revealed that circARRDC 3 regulated AR progression by targeting miR-375 in IL-13-induced NECs.

Overexpression of KLF4 reverses the miR-375-mediated inhibitory effects on AR development. To investigate the potential effects of miR-375 in NECs, bioinformatics tools (TargetScan) were used to predict the possible targets. It was found that the 3'-UTR of KLF4 had direct binding sites for miR-375 (Fig. 3A). In addition, the luciferase reporter assay indicated that transfection with the miR-375 mimic decreased and the miR-375 inhibitor increased the luciferase activity of KLF4-WT, while there were no changes observed in the KLF4-Mut group (Fig. 3B). Moreover, RT-qPCR and western blotting revealed that miR-375 upregulation decreased KLF4 expression and miR-375 downregulation increased KLF4 expression (Fig. 3C and D). Collectively, the results suggested that miR-375 could directly interact with KLF4 and significantly inhibit its expression.

To further assess the roles of KLF4 and miR-375 in AR, NC mimic, miR-375 mimic, and miR-375 mimic + KLF4 were transfected into IL-13-induced NECs. RT-qPCR showed 
A

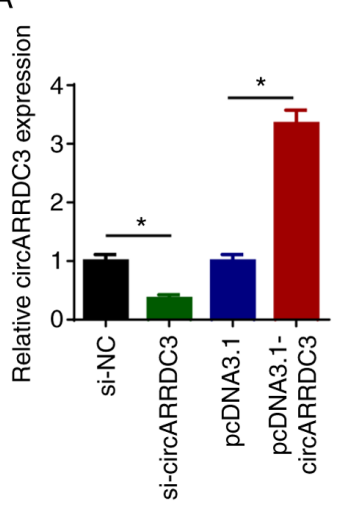

B

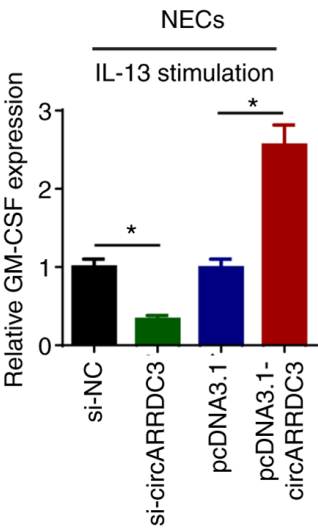

C

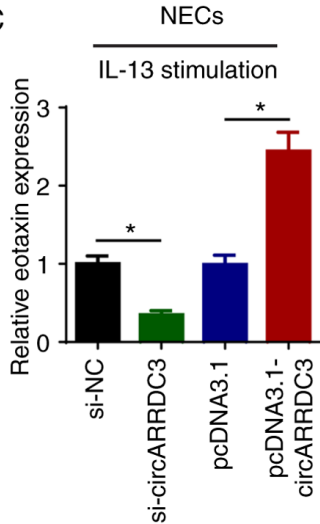

F

E

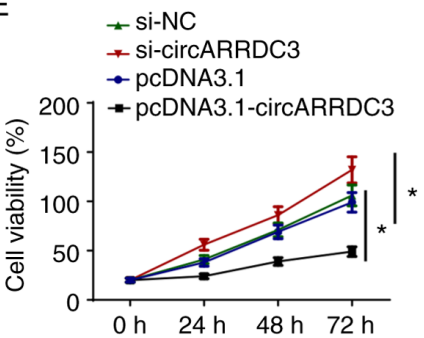

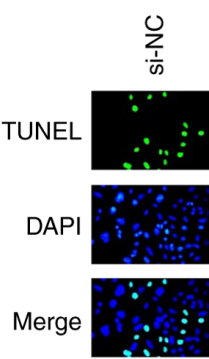

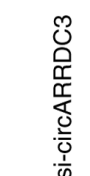

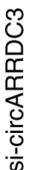
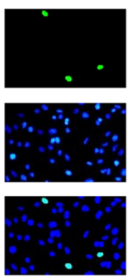

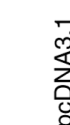

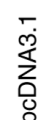

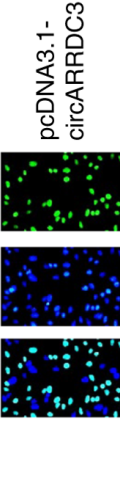

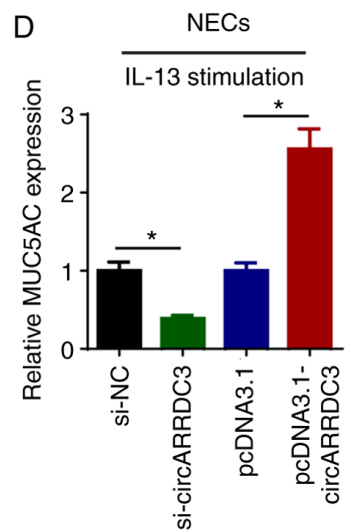

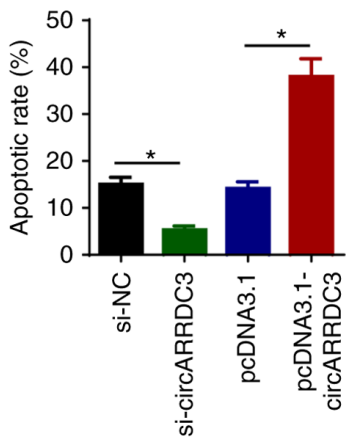

Figure 1. circARRDC3 regulates IL-13-induced inflammatory cytokines, mucus production, cell viability and apoptosis of NECs. (A) circARRDC3 expression levels were detected by RT-qPCR in NECs transfected with si-NC, si-circARRDC3, pcDNA3.1 and pcDNA3.1-circARRDC3. The levels of (B) GM-CSF, (C) eotaxin and (D) MUC5AC were detected by RT-qPCR in IL-13-induced NECs transfected with si-NC, si-circARRDC3, pcDNA3.1 and pcDNA3.1-circARRDC3. (E) Cell viability was examined by an MTT assay in IL-13-induced NECs transfected with si-NC, si-circARRDC3, pcDNA3.1 and pcDNA3.1-circARRDC3. (F) Cell apoptosis was examined using a TUNEL assay in IL-13-induced NECs transfected with si-NC, si-circARRDC3, pcDNA3.1 and pcDNA3.1-circARRDC3. Magnification, x100. "P<0.05. ARRDC3, arrestin domain-containing 3; circ-, circular RNA; NEC, nasal epithelial cell; RT-qPCR, reverse transcription-quantitative PCR; si-, small interfering RNA; NC, negative control; GM-CSF, granulocyte-macrophage colony-stimulating factor; MUC5AC, mucin 5AC.

the KLF4 expression was significantly increased in NECs transfected with pcDNA3.1-KLF4 (Fig. 3E). As shown in Fig. 3F-H, KLF4 overexpression reversed the suppressive effects of miR-375 mimic transfection on the expression levels of GM-CSF, eotaxin and MUC5AC. In addition, the upregulation of miR-375 promoted cell viability in IL-13-induced NECs, while these effects were abolished by KLF4 overexpression (Fig. 3I). Furthermore, miR-375 mimic inhibited apoptosis of IL-13-induced NECs, which was partially reversed following KLF4 overexpression (Fig. 3J). The results indicated that miR-375 expression negatively regulated KLF4 expression by directly interacting with each other in IL-13-induced NECs.

circARRDC3, miR-375 and KLF4 are dysregulated in $A R$ tissues. RT-qPCR showed that circARRDC3 and KLF4 were increased, and miR-375 was decreased in AR tissues (Fig. 4A-C). Furthermore, miR-375 expression was negatively correlated with circARRDC3 or KLF4 expression. Whereas, circARRDC3 expression was positively correlated with KLF4 expression in AR tissues (Fig. 4D-F). To summarize, circARRDC3, miR-375 and KLF4 were dysregulated in the clinical nasal mucosa of patients with AR.

\section{Discussion}

Increasing evidence has indicated that circRNAs are involved in inflammatory activities of several diseases $(23,24)$. For example, Qin et al (25) indicated that circRNA-9119 suppressed polyriboinosinic polyribocytidylic acid-induced inflammation in Leydig and Sertoli cells via toll-like receptor 3 and antiviral innate immune response receptor RIG-I signalling pathways. Yang et al (26) reported that circ_0000950 promoted neuronal apoptosis and increased inflammatory cytokine levels in Alzheimer's disease. To the best of our knowledge, no published studies have investigated the association between circARRDC3 and AR. The present study observed that circARRDC3 knockdown significantly suppressed levels of GM-CSF, eotaxin and MUC5AC, and promoted cell viability in IL-13-induced NECs, suggesting that circARRDC3 promoted AR development.

Recent studies have indicated that circRNAs could serve as miRNA sponges to suppress miRNA expression, and thereby suppress miRNA function in inflammatory disease. For example, Ye et al (27) reported that dysregulation of circRNA_103516 participated in the molecular mechanism of inflammatory bowel disease by regulating miR-19b-1-5p. 
Mut circARRDC3 : 5' AC-TGA-CT-AGGAGGAGG $3^{\prime}$

WT circARRDC3 : 5' AC-TGA-CT-AGGGAACAA 3' | || || |

hsa-miR-375 : $\quad$ 3' TGCGCTCGGCTTGCTTGTT 5

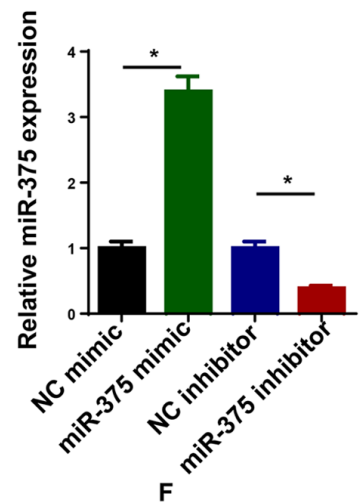

C

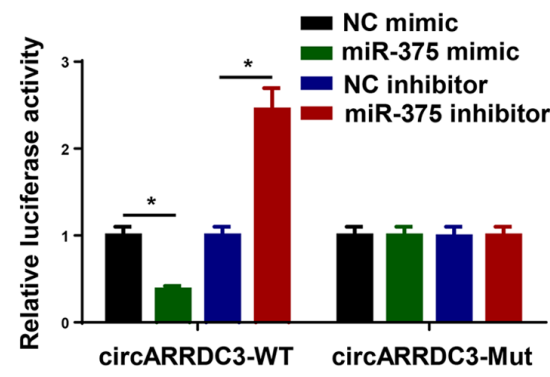

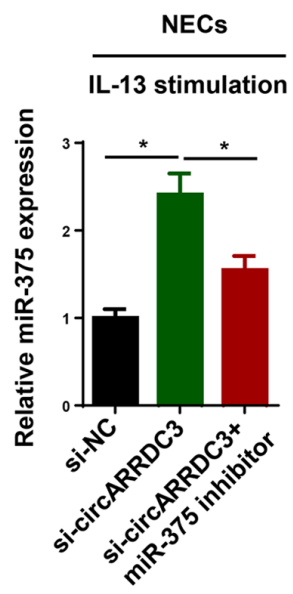

E

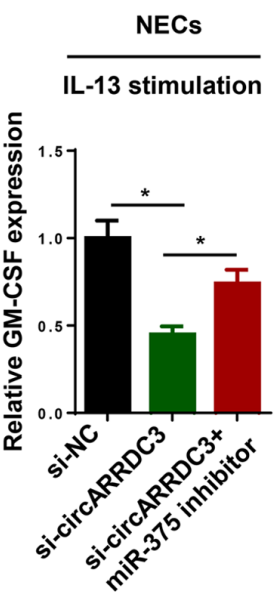

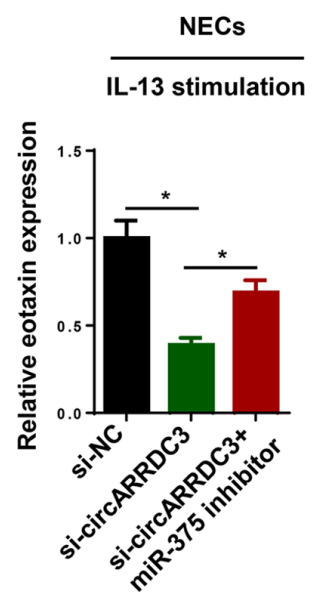

G

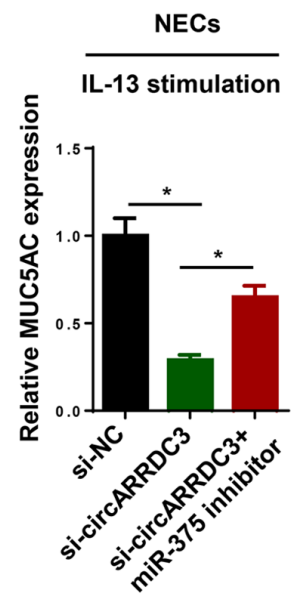

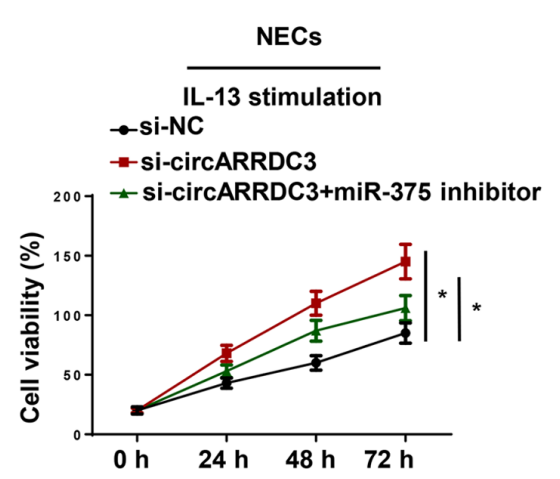

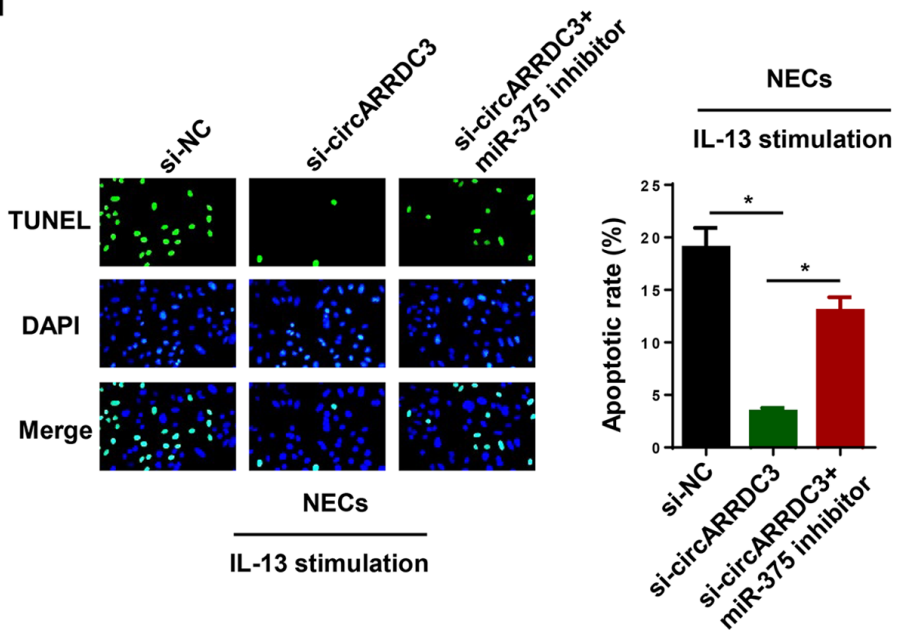

Figure 2. miR-375 interacts with circARRDC3. (A) Bioinformatics prediction of putative binding site at circARRDC3 by miR-375. (B) RT-qPCR was performed to determine the expression of miR-375 in NECs transfected with miR-375 mimic or miR-375 inhibitor. (C) Dual luciferase reporter assay showed luciferase activity in WT circARRDC3-containing NECs transfected with NC mimic, miR-375 mimic, NC inhibitor and miR-375 inhibitor. (D) miR-375 expression levels were detected by RT-qPCR in IL-13-induced NECs transfected with si-NC, si-circARRDC3, and si-circARRDC3 + miR-375 inhibitor. The levels of (E) GM-CSF, (F) eotaxin (G) and MUC5AC were detected by RT-qPCR in IL-13-induced NECs transfected with si-NC, si-circARRDC3, and si-circARRDC3 + miR-375 inhibitor. (H) Cell viability was examined by an MTT assay in IL-13-induced NECs transfected with si-NC, si-circARRDC3, and si-circARRDC3 + miR-375 inhibitor. (I) Cell apoptosis was examined using a TUNEL assay in IL-13-induced NECs transfected with si-NC, si-circARRDC3, si-circARRDC3 + miR-375 inhibitor. Magnification, x100. ${ }^{*}$ P $<0.05$. ARRDC3, arrestin domain-containing 3; circ-, circular RNA; NEC, nasal epithelial cell; RT-qPCR, reverse transcription-quantitative PCR; si-, small interfering RNA; NC, negative control; GM-CSF, granulocyte-macrophage colony-stimulating factor; MUC5AC, mucin 5AC; miR, microRNA; WT, wild-type; Mut, mutant.

Zhang et al (28) demonstrated that circARF3 acted as an endogenous miR-103 sponge to inhibit mitophagy-mediated adipose inflammation both in vitro and in vivo. Xue et al (29) indicated that circ_0000638 suppressed neodymium oxide-induced bronchial epithelial cell inflammation via the miR-498-5p/NF- $\kappa$ B axis. The present study found that
miR-375 is a downstream target of circARRDC3. Knockdown of circARRDC3 suppressed inflammatory cytokines, mucus production and cell apoptosis in IL-13-induced NECs, which was reversed following miR-375 inhibitor transfection. In addition, the effects of circARRDC3 knockdown on promoting the viability of IL-13-induced NECs were abolished by decreasing 
A

Mut KLF4 : 5 ' AguaugccuUaAgCagccaggg $3^{\prime}$

WT KLF4 : 5' AGUAUGCCUUAAGCAGAACAAA 3'

|| ||||

hsa-miR-375 : 3' AGUGCGCUCGGCUUGCUUGUUU 5'

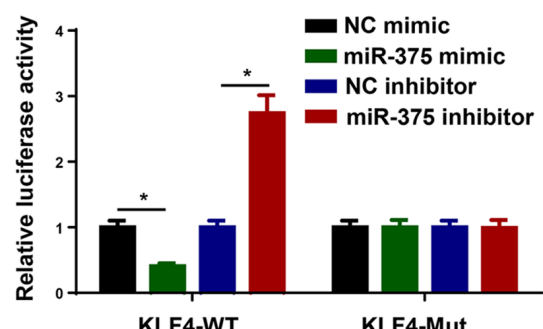

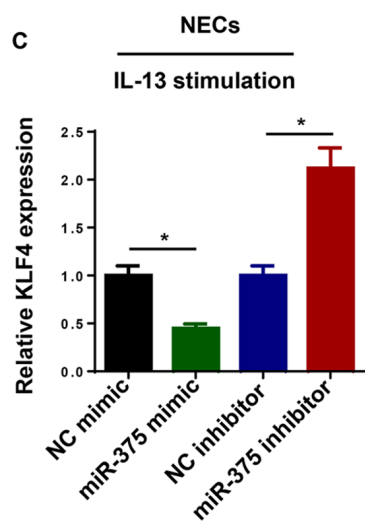

D
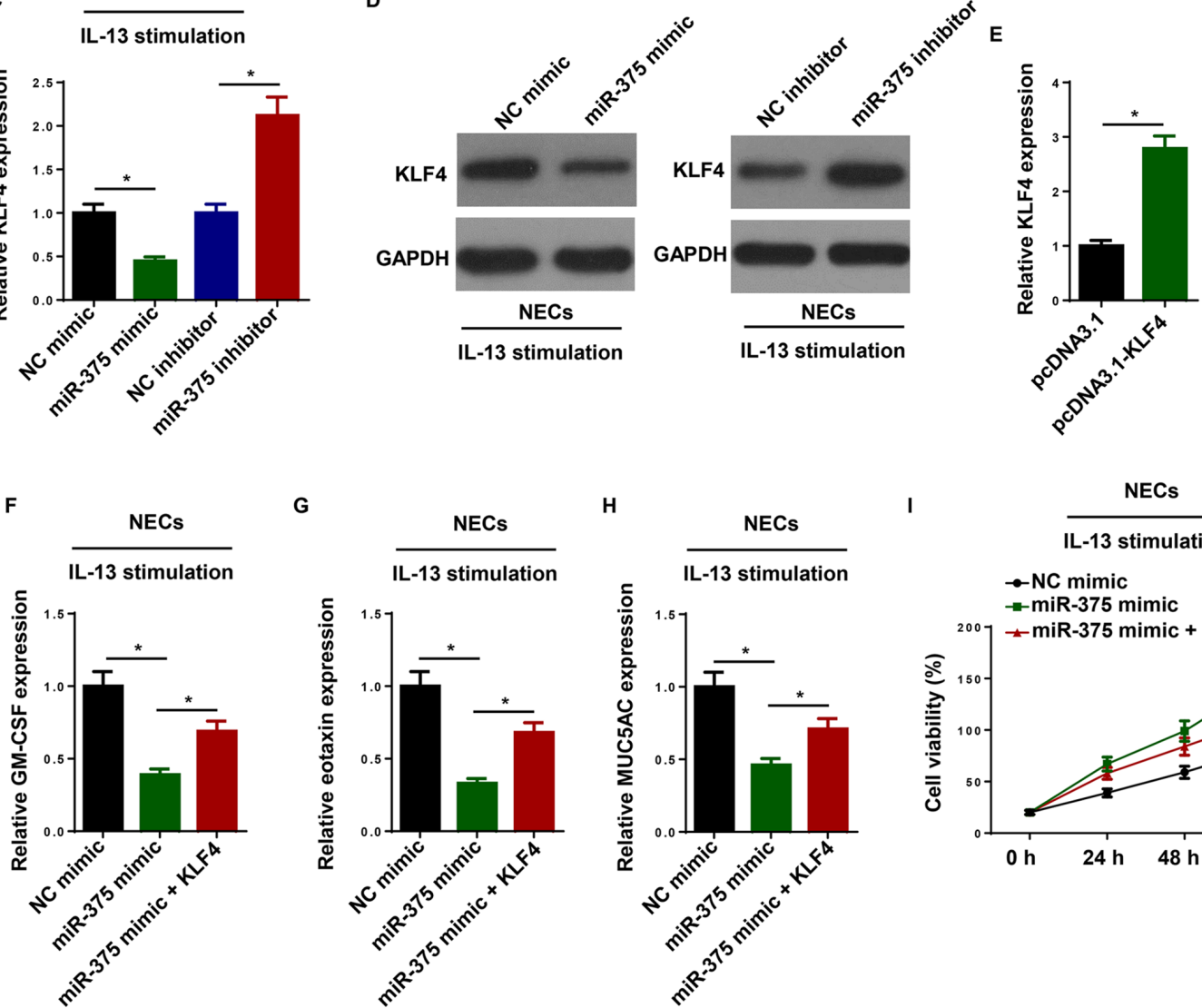

G

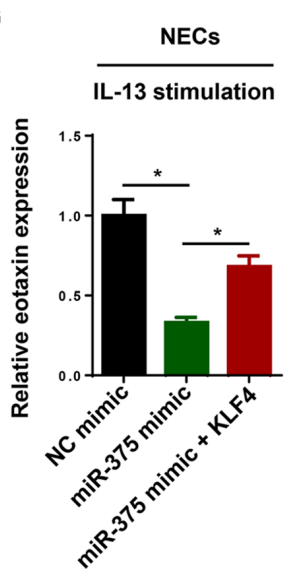

H

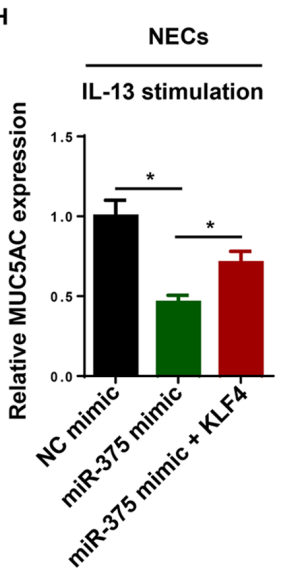

1

NECs

IL-13 stimulation
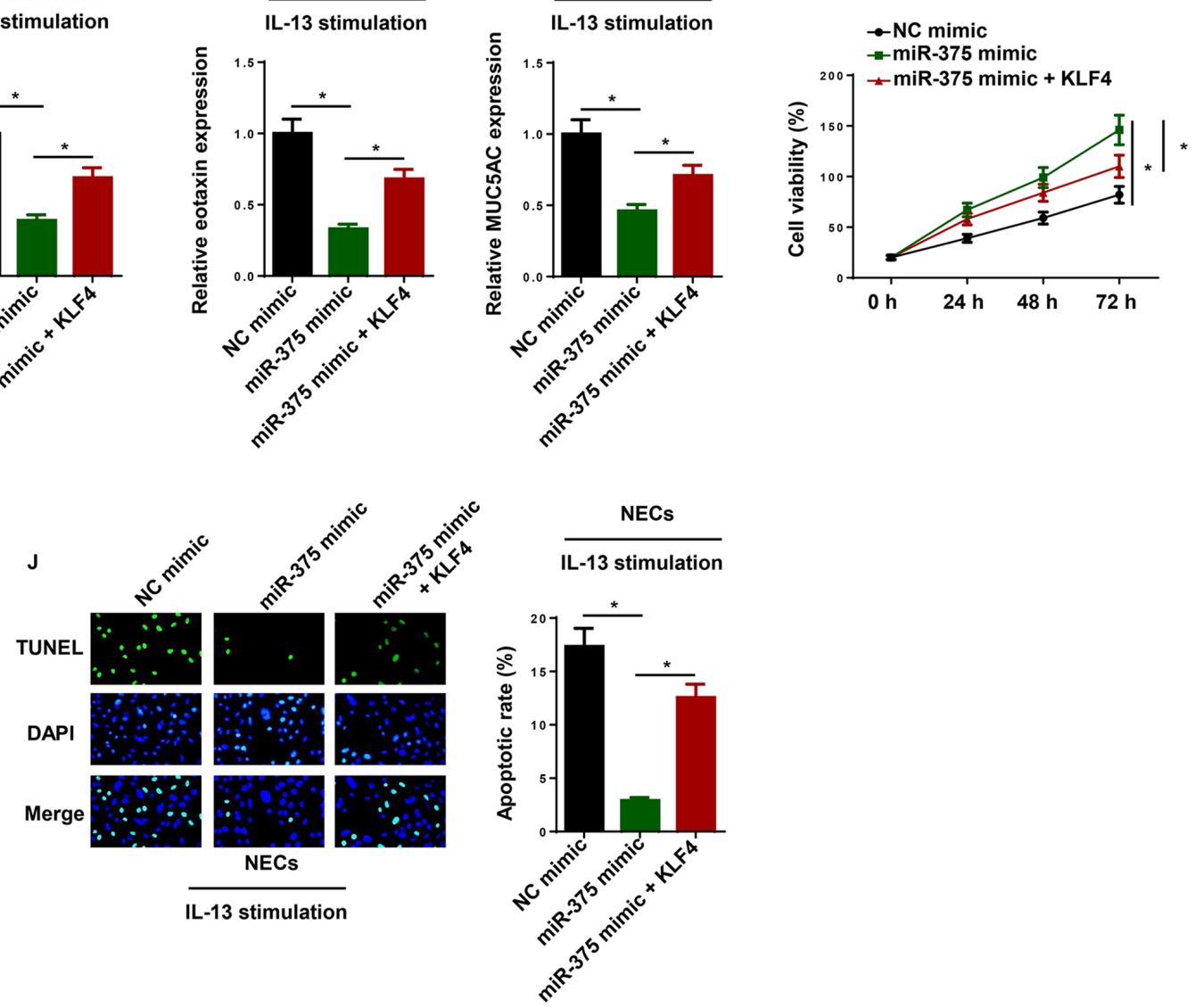

Figure 3. Overexpression of KLF4 reverses miR-375-mediated inhibitory effects on AR development. (A) Bioinformatics prediction of putative binding site at 3'-UTR of KLF4 by miR-375. (B) Dual luciferase reporter assay showed luciferase activity in WT KLF4-containing NECs transfected with NC mimic, miR-375 mimic, NC inhibitor and miR-375 inhibitor. The levels of KLF4 were detected by (C) RT-qPCR and (D) western blotting in IL-13-induced NECs transfected with NC mimic, miR-375 mimic, NC inhibitor and miR-375 inhibitor. (E) RT-qPCR was performed to determine KLF4 expression in NECs transfected with pcDNA3.1 and pcDNA3.1-KLF4. The levels of (F) GM-CSF, (G) eotaxin and (H) MUC5AC were detected by RT-qPCR in IL-13-induced NECs transfected with NC mimic, miR-375 mimic, and miR-375 mimic + KLF4. (I) Cell viability was examined by an MTT assay in IL-13-induced NECs transfected with NC mimic, miR-375 mimic, and miR-375 mimic + KLF4. (J) Cell apoptosis was examined using a TUNEL assay in IL-13-induced NECs transfected with NC mimic, miR-375 mimic, and miR-375 mimic + KLF4. Magnification, x100. "P<0.05. NEC, nasal epithelial cell; RT-qPCR, reverse transcription-quantitative PCR; NC, negative control; GM-CSF, granulocyte-macrophage colony-stimulating factor; MUC5AC, mucin 5AC; miR, microRNA; WT, wild-type; Mut, mutant; KLF4, krueppel-like factor 4; UTR, untranslated region. 
A
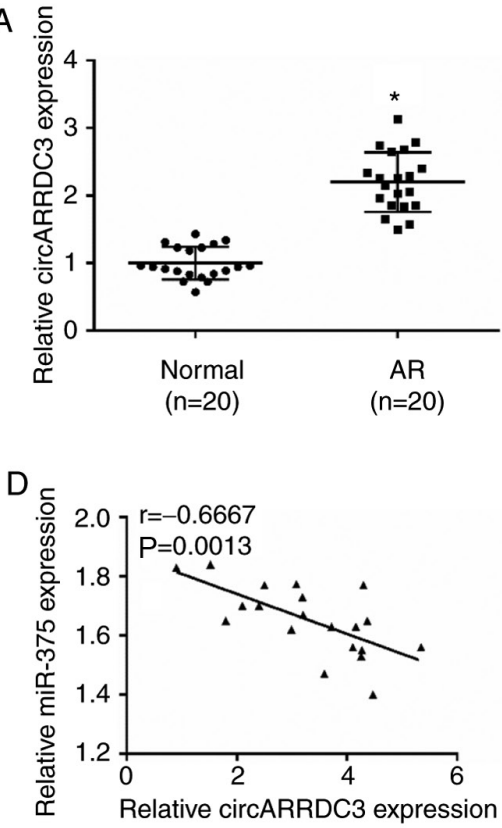
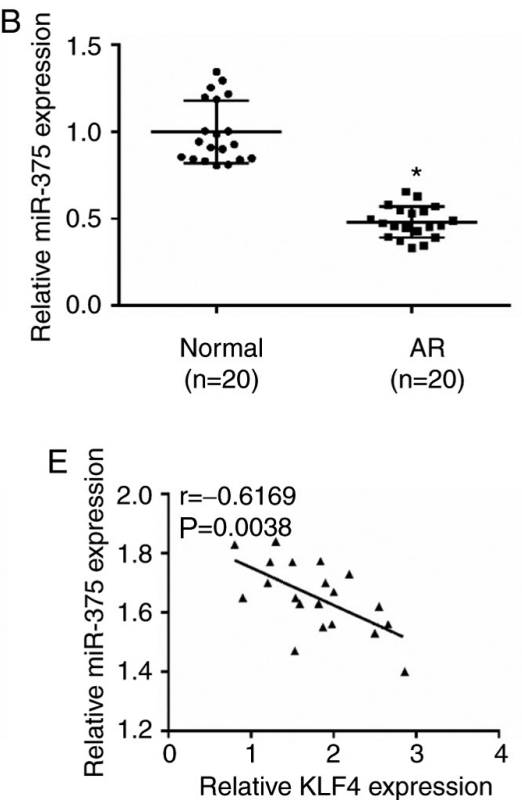
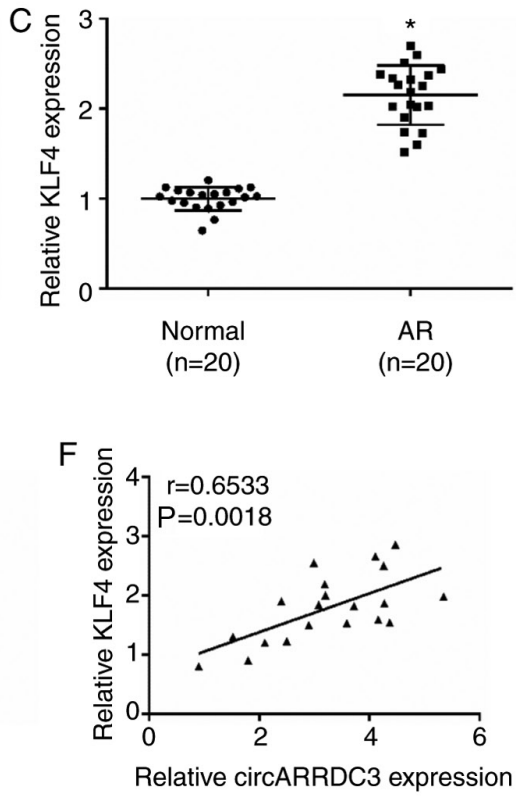

Figure 4. circARRDC3, miR-375 and KLF4 are dysregulated in AR tissues. (A) circARRDC3, (B) miR-375 and (C) KLF4 expression levels were detected by reverse transcription-quantitative PCR in nasal mucosa of normal healthy subjects $(n=20)$ and patients with AR (n=20). (D) Pearson's correlation analysis indicated a negative correlation between circARRDC3 and miR-375 expression in the nasal mucosa of patients with AR ( $\mathrm{n}=20$ ). (E) Pearson's correlation analysis indicated a negative correlation between KLF4 and miR-375 expression in the nasal mucosa of patients with AR ( $\mathrm{n}=20$ ). (F) Pearson's correlation analysis indicated a positive correlation between circARRDC3 and KLF4 expression in the nasal mucosa of patients with AR ( $\mathrm{n}=20$ ). ${ }^{*} \mathrm{P}<0.05$ vs. normal healthy subjects. ARRDC3, arrestin domain-containing 3; circ-, circular RNA; miR, microRNA; KLF4, krueppel-like factor 4; AR, allergic rhinitis.

miR-375 expression. These results indicated that circARRDC3 regulated the development of AR via targeting miR-375.

Our previous study showed that miR-375 was significantly downregulated in AR mice and significantly ameliorated AR via the JAK2/STAT3 pathway (30). Considering the comprehensive mechanisms underlying AR progression, we further investigated several AR-related genes, including KLF4. KLF4 is a transcription factor required for epithelial-mesenchymal transition and establishes the skin barrier $(31,32)$. Moreover, Kurotaki et al (33) revealed that KLF4 is essential for murine monocyte differentiation. Using bioinformatics analysis, Liu et al (34) found that KLF4 was significantly upregulated in patients with seasonal allergic rhinitis. Mao et al (35) found a direct association between miR-375 and KLF4, which prompted us to assess the assosciation between miR-375 and KLF4 and their potential effects in AR progression and development. The present study found that miR-375 could directly bind to KLF4 in AR tissues. In addition, KLF4 overexpression abolished the suppressive effects of miR-375 mimic on GM-CSF, eotaxin and MUC5AC expression levels and apotosis in IL-13-induced NECs. Moreover, KLF4 overexpression reversed the miR-375 mimic-induced effects on cell viability. To the best of our knowledge, the present study was the first to demonstrate that circARRDC3 promoted IL-13-induced inflammatory cytokine and mucus production in NECs via the miR-375/KLF4 axis. In addition, miR-375 was negatively correlated with circARRDC3 and KLF4 expression in AR tissues, whereas circARRDC3 expression was positively correlated with KLF4 expression.

In conclusion, the present study demonstrated that circARRDC3 promoted AR progression by regulating the miR-375/KLF4 axis, providing a potential therapeutic target for the treatment of AR. This study primarily focused on the role of circARRDC3 in IL-13-induced NECs in vitro. Therefore, the function of circARRDC3 in the primary tissues of patients with AR and in animal experiments will be investigated in the future.

\section{Acknowledgements}

Not applicable.

\section{Funding}

No funding was received.

\section{Availability of data and materials}

The datasets used and/or analyzed during the present study are available from the corresponding author upon reasonable request.

\section{Authors' contributions}

TW and PW conceived and designed the study. DC, ZX, and LY contributed to the acquisition, analysis, and interpretation of data. TW and PW drafted and revised the manuscript. All authors read and approved the final manuscript.

\section{Ethics approval and consent to participate}

The present study was approved by the ethics committee of the Shanghai Ninth People's Hospital (Shanghai, China). Written informed consent was obtained from all patients. 


\section{Patient consent for publication}

Not applicable.

\section{Competing interests}

The authors declare that they have no competing interests.

\section{References}

1. Ozdoganoglu T and Songu M: The burden of allergic rhinitis and asthma. Ther Adv Respir Dis 6: 11-23, 2012.

2. Bauchau V and Durham SR: Epidemiological characterization of the intermittent and persistent types of allergic rhinitis. Allergy 60: 350-353, 2005.

3. Leynadier F, Mees K, Arendt C and Pinelli ME: Efficacy and safety of levocetirizine in seasonal allergic rhinitis. Acta Otorhinolaryngol Belg 55: 305-312, 2001.

4. Wang DY: Risk factors of allergic rhinitis: Genetic or environmental? Ther Clin Risk Manag 1: 115-123, 2005.

5. Memczak S, Jens M, Elefsinioti A, Torti F, Krueger J, Rybak A, Maier L, Mackowiak SD, Gregersen LH, Munschauer M, et al: Circular RNAs are a large class of animal RNAs with regulatory potency. Nature 495: 333-338, 2013.

6. Liu J and Cao Z: Protective Effect of Circular RNA (CircRNA) Ddx17 in Ovalbumin (OVA)-Induced Allergic Rhinitis (AR) Mice. Med Sci Monit 26: e919083, 2020.

7. Zhu X, Wang X, Wang Y and Zhao Y: The regulatory network among CircHIPK3, LncGAS5, and miR-495 promotes Th2 differentiation in allergic rhinitis. Cell Death Dis 11: 216, 2020.

8. Yue L, Yin X, Hao F, Dong J, Ren X, Xu O and Shan C: Long noncoding RNA Linc00632 inhibits interleukin-13-induced inflammatory cytokine and mucus production in nasal epithelial cells. J Innate Immun 12: 116-128, 2020.

9. Shin IS, Lee MY, Jeon WY, Shin NR, Seo CS and Ha H: EBM84 attenuates airway inflammation and mucus hypersecretion in an ovalbumin-induced murine model of asthma. Int J Mol Med 31: 982-988, 2013.

10. Wang L, Lv Q, Song X, Jiang K and Zhang J: ADRB2 suppresses IL-13-induced allergic rhinitis inflammatory cytokine regulated by miR-15a-5p. Hum Cell 32: 306-315, 2019.

11. Carleton M, Cleary MA and Linsley PS: MicroRNAs and cell cycle regulation. Cell Cycle 6: 2127-2132, 2007.

12. Chen S, Liu Y, Wang Y and Xue Z: LncRNA CCAT1 promotes colorectal cancer yumorigenesis via a miR-181b-5p/TUSC3 axis OncoTargets Ther 12: 9215-9225, 2019

13. Teng Y, Zhang R, Liu C, Zhou L, Wang H, Zhuang W, Huang Y and Hong $\mathrm{Z}$ : miR-143 inhibits interleukin-13-induced inflammatory cytokine and mucus production in nasal epithelial cells from allergic rhinitis patients by targeting IL13R $\alpha 1$. Biochem Biophys Res Commun 457: 58-64, 2015.

14. Liu HC, Liao Y and Liu CQ: miR-487b mitigates allergic rhinitis through inhibition of the IL-33/ST2 signaling pathway. Eur Rev Med Pharmacol Sci 22: 8076-8083, 2018.

15. Chen RF, Huang HC, Ou CY,Hsu TY, Chuang H, Chang JC, Wang L, Kuo HC and Yang KD: MicroRNA-21 expression in neonatal blood associated with antenatal immunoglobulin E production and development of allergic rhinitis. Clin Exp Allergy 40: 1482-1490, 2010.

16. Dissanayake E and Inoue Y: MicroRNAs in allergic disease Curr Allergy Asthma Rep 16: 67, 2016.

17. Ingram JL and Kraft M: IL-13 in asthma and allergic disease: Asthma phenotypes and targeted therapies. J Allergy Clin Immunol 130: 829-842, quiz 843-844, 2012.

18. Lv J, Xiong Y, Li W, Cui X, Cheng X, Leng Q and He R: IL-37 inhibits IL-4/IL-13-induced CCL11 production and lung eosinophilia in murine allergic asthma. Allergy 73: 1642-1652, 2018.
19. Ashraf MI, Shahzad M and Shabbir A: Oxyresveratrol ameliorates allergic airway inflammation via attenuation of IL-4, IL-5, and IL-13 expression levels. Cytokine 76: 375-381, 2015.

20. Zhu X, Wang X, Wang Y and Zhao Y: Exosomal long non-coding RNA GAS5 suppresses Th1 differentiation and promotes Th2 differentiation via downregulating $\mathrm{EZH} 2$ and T-bet in allergic rhinitis. Mol Immunol 118: 30-39, 2020.

21. Agarwal V, Bell GW, Nam JW and Bartel DP: Predicting effective microRNA target sites in mammalian mRNAs. eLife 4: 4, 2015.

22. Livak KJ and Schmittgen TD: Analysis of relative gene expression data using real-time quantitative PCR and the 2(-Delta Delta C(T)) method. Methods 25: 402-408, 2001.

23. Hwang JY, Kim EB, Ka H and Lee CK: Identification of the porcine XIST gene and its differential $\mathrm{CpG}$ methylation status in male and female pig cells. PLoS One 8: e73677, 2013.

24. Shenoda BB, Tian Y, Alexander GM, Aradillas-Lopez E, Schwartzman RJ and Ajit SK: miR-34a-mediated regulation of XIST in female cells under inflammation. J Pain Res 11: 935-945, 2018.

25. Qin L, Lin J and Xie X: CircRNA-9119 suppresses poly I:C induced inflammation in Leydig and Sertoli cells via TLR3 and RIG-I signal pathways. Mol Med 25: 28, 2019.

26. Yang H, Wang H, Shang H, Chen X, Yang S, Qu Y, Ding J and Li X: Circular RNA circ 0000950 promotes neuron apoptosis, suppresses neurite outgrowth and elevates inflammatory cytokines levels via directly sponging miR-103 in Alzheimer's disease. Cell Cycle 18: 2197-2214, 2019.

27. Ye YL, Yin J, Hu T, Zhang LP, Wu LY and Pang Z: Increased circulating circular RNA 103516 is a novel biomarker for inflammatory bowel disease in adult patients. World J Gastroenterol 25 6273-6288, 2019.

28. Zhang Z, Zhang T, Feng R, Huang H, Xia T and Sun C: circARF3 alleviates mitophagy-mediated inflammation by targeting miR-103/TRAF3 in mouse adipose tissue. Mol Ther Nucleic Acids 14: 192-203, 2019.

29. Xue H, Yu F, Zhang X, Liu L and Huang L: circ_0000638 inhibits neodymium oxide-induced bronchial epithelial cell inflammation through the miR-498-5p/NF- $\kappa \mathrm{B}$ axis. Ecotoxicol Environ Saf 195: 110455, 2020

30. Wang T, Chen D, Wang P, Xu Z and Li Y: miR-375 prevents nasal mucosa cells from apoptosis and ameliorates allergic rhinitis via inhibiting JAK2/STAT3 pathway. Biomed Pharmacother 103: 621-627, 2018

31. Segre JA, Bauer C and Fuchs E: Klf4 is a transcription factor required for establishing the barrier function of the skin. Nat Genet 22: 356-360, 1999.

32. Tiwari N, Meyer-Schaller N, Arnold P, Antoniadis H, Pachkov M, van Nimwegen $\mathrm{E}$ and Christofori G: Klf4 is a transcriptional regulator of genes critical for EMT, including Jnk1 (Mapk8). PLoS One 8: e57329, 2013.

33. Kurotaki D, Osato N, Nishiyama A, Yamamoto M, Ban T, Sato H, Nakabayashi J, Umehara M, Miyake N, Matsumoto N, et al: Essential role of the IRF8-KLF4 transcription factor cascade in murine monocyte differentiation. Blood 121: 1839-1849, 2013.

34. Liu Y, Shi J and Chen X: Identification of novel targets for seasonal allergic rhinitis during and outside the pollen season by microarray analysis. Acta Otolaryngol 135: 1330-1336, 2015.

35. Mao Q, Quan T, Luo B, Guo X, Liu L and Zheng Q: MiR-375 targets KLF4 and impacts the proliferation of colorectal carcinoma. Tumour Biol 37: 463-471, 2016.

This work is licensed under a Creative Commons Attribution-NonCommercial-NoDerivatives 4.0 International (CC BY-NC-ND 4.0) License. 